\title{
Control Decision Rule for European Chafer (Coleoptera: Scarabaeidae) Larvae Infesting Turfgrass
}

\author{
J. P. NYROP, M. G. VILLANI, AND J. A. GRANT ${ }^{1}$ \\ Department of Entomology, Cornell University, New York State Agricultural Experiment Station, \\ Geneva, NY 14456
}

\begin{abstract}
Environ. Entomol. 24(3): 521-528 (1995)
ABSTRACT A control decision rule for European chafer, Rhizotrogus (Amphimallon) majalis (Razoumowsky), larvae infesting turfgrass was developed using data from 317 residential sites. Larvae were often abundant enough to cause damage to turf in portions (patches) of properties when average density over an entire site was much less than a damage threshold of $5-10$ grubs per $950 \mathrm{~cm}^{2}$. To account for this, an empirical relationship between the size of the largest patch of European chafer larvae at a site and site-wide density was used in the development of the decision rule. Properties with a patch of grubs in excess of $\approx 30 \mathrm{~m}^{2}$ were deemed to require insecticide treatment. Site characteristics (lawn age, shade, and percentage of Kentucky bluegrass) were related to site-wide density and this relationship was used to formulate a risk assessment system. This system is used to determine whether a site should be sampled or not. Properties that are not sampled are not to be treated. Other treatment decisions are based on the outcome of sampling. The relationship between site-wide: density and patch size did not allow clear identification of a density that could be used as a threshold in a sampling program. Therefore, several sampling plans were constructed that classified density according to different threshold values. Operating characteristic functions were used in combination with the aforementioned relationship between density and patch size to calculate two types of errors for each sampling plan: the probability of not treating when treatment was necessary and the probability of treating when treatment was not required. Based on these error functions, a threshold of 0.25 grubs per $11-\mathrm{cm}$ diameter turf plug was advocated. Use of the proposed control decision rule should result in few treatment errors but could lead to considerable reductions in pesticide use.
\end{abstract}

KEY WORDS sampling, decision making, turf pests

IN THE NORTHEASTERN United States, turfgrass is attacked by a complex of scarab species including the Japanese beetle, Popillia japonica Newman, the European chafer, Rhizotrogus (Amphimallon) majalis (Razoumowsky), and the Oriental beetle Exomala orientalis (Tashiro 1987). These scarab grubs can be controlled by one well-timed insecticide application; however, one or two treatments are usually made prophylactically or in response to turf damage. Despite the existence of damage thresholds (Tashiro 1987, Shetlar et al. 1990) grub populations are seldom assessed before treatment decisions are made.

Pest control decision rules (Binns \& Nyrop 1992) can be used to rationally schedule pesticide use. These protocols consist of at least two components and may include a third: a procedure for assessing the density of the pest population, an economic or action threshold, and a phenological forecast which is often needed to determine the appropriate time to measure population density.

'Integrated Pest Management Program, Cornell University, New York State Agricultural Experiment Station, Geneva, NY 14456 .
By using effective pest control decision rules, managers can restrict the application of pesticides to those situations where high pest densities warrant their use. This is especially important when considering control of turfgrass pests because indiscriminate and excessive use of pesticides might expose large numbers of humans to biologically active chemicals.

Even though there is a clear need for control decision rules for scarab larvae in turf, none are currently in use and little work has been devoted toward developing such protocols. There are only three published articles that report on this subject. Burrage \& Gyrisco (1954) found populations of European chafer grubs to be clumped in pastures in New York and determined that square-foot diggings were more efficient than larger sampling units. Ng et al. (1983a, b) fit negative binomial distribution models to counts of Japanese beetle grubs in square-foot sample units and constructed a sequential classification sampling plan based on these models.

The information these studies convey is not sufficient for developing control decision rules for 
scarab grubs. At first glance this is not obvious. Pest management decision rules are usually based on classifying density with respect to a threshold using sampling plans constructed by characterizing sampling experiments by way of empirical or probability distribution models (Nyrop \& Binns 1991). Economic thresholds for scarab grubs in turfgrass are generally considered to be 5-10 per $950 \mathrm{~cm}^{2}$ (Tashiro 1987). Knowing this and the sampling distribution for counts of scarab grubs, it would seem to be very straightforward to construct a control decision rule. However, scarab grubs are patchily distributed and it is often the case that when the number of larvae exceed densities that cause damage, they do so only in portions of a site and the overall mean density is much below a damage threshold. Because it is necessary to control larvae in patches with high density, mean density by itself is not a suitable parameter for use in a control decision rule. Although it is certainly possible to sample a site sufficiently to map patches with high grub densities, this is too costly for wide scale use. $\AA$ different approach would be to use data from a site to indicate whether the property was likely to harbor patches with high grub densities and treat the entire site accordingly. This has the disadvantage that entire sites are treated when only a portion of the site requires control. However, provided that only a modest portion of sites require any control at all, use of this type of decision rule would still lead to greatly improved pesticide use compared with prophylactic treatments. Furthermore, such a rule might actually be used by practitioners, whereas decision rules that require intensive mapping of grub densities will almost certainly be shumned because of the high cost of their in:plementation. In this article we describe a pest control decision rule for European chafer that is based on the paradigm of using site-specific data to indicate whether high density patches of grubs are likely to be present.

\section{Materials and Methods}

We collected data from 317 residential lawns near Rochester, NY, for use in formulating the decision rule. Two types of data were collected at each site; counts of grubs along a grid placed over the property and estimates of variables that described the site. Sites were sampled during the lst and 2nd wk of September 1990 when European chafer were in the third instar.

European chafer grub densities were mapped at: each site by counting grubs in samples collected at regular intervals from throughout the property. Samples were located along a grid with $3 \mathrm{~m}$ between sample locations. Each sample consisted of 11-cm diameter plugs cut from the turf to a depth of $10 \mathrm{~cm}$. All grubs in the soil were collected by removing soil to this depth. The $11-\mathrm{cm}$ plugs were used in lieu of the more conventional square-foot sample unit because preliminary studies showed the plugs were easier to collect, provided as much sample information as the square-foot sample unit, and caused less damage to the turf. The number of samples per site ranged from 20 to over 180 , depending on the size of the property.

When the grubs were sampled, sites were described by determining lawn age ( $\leq 20$ or $>20 \mathrm{yr}$ ) and estimating grass species composition, soil type, slope, thatch content, and percentage of shading. Values for each of the variables other than lawn age were categorical estimates subjectively selected from one of three possible choices. We purposefully collected these data in this apparently casual manner because we wished the data to be representative of what lawn care specialists would be able to collect. Percentage of shade $(<30,30-$ 60 , or $>60 \%$ ) and slope (level, sloping, or low lying) were estimated by overall assessment of the property. Grass species composition ( $<30,30-60$, and $>60 \%$ ) was determined by examining the turf at five or more sites on the property; however, variability among sites and the numbers of samples taken were not recorded. The amount of thatch present $(<1.5,1.5-2.5$, or $>2.5 \mathrm{~cm})$ and soil type (clay, loam, or sand) was determined by examining five or more cores removed from the soil profile.

The control decision rule was developed using the following four steps: first, counts of grubs were used to determine whether there was a relationship between average grub density and the size of patches of grubs as well as the density of grubs in a patch. From preliminary examination of the data we knew that there were often cases where the average density of grubs was low, but there existed a smaller area on the property (a patch) where grubs were relatively abundant. If a relationship between property-wide density and density in a patch or patch size, or both existed, we intended to exploit this pattern in the decision rule.

In the second step, site description data were used to determine whether there was a relationship between some or all of these variables and site-wide grub density. If such a relationship occurred we planned to use site characteristics to classify a property as either requiring sampling or not requiring sampling. If no samples were needed, no pesticide would be applied for control of European chafer. If sampling was required, the decision to treat or not treat the site for European chafers would depend on the outcome of sampling.

Next, counts of grubs were used to construct models that could be used to describe sampling experiments. These models are required to assess the performance of sampling plans used in control decision rules (Nyrop \& Binns 1991) and consisted of Taylor's (1961) variance-mean model and Poisson and negative binomial probability models.

Finally, control decision rules based on double sampling plans (Nyrop \& Wright 1985) were formulated and their performance was evaluated. Each of these steps will now be described in more detail. 
Density-Patch Relationship. European chafer larvae are capable of causing economic injury to turf when their density exceeds $5-10$ per $950 \mathrm{~cm}^{2}$ (Tashiro 1987). This translates to $\approx 0.5-1$ grub per 11-cm plug. When we began to examine the sample data, it quickly became apparent that there were areas of lawns where average European chafer larval density exceeded one per plug; however, the average density throughout the property was much less than one per plug. From a lawn care perspective, it is important to treat patches of turf in which European chafer grub density exceeds one per 11-cm plug. Based on our experience, we defined a patch of European chafer larvae necessitating treatment to be four or more contiguous sample locations having one or more larvae per plug. Thus, with our definition, properties with European chafer populations requiring control were those where four or more adjacent sample sites yielded one or more chafer larvae. As a result, a control decision rule for European chafer larvae infesting residential lawns must be able to accurately and parsimoniously identify properties where larval densities exceed one per $11-\mathrm{cm}$ plug in patches of $27 \mathrm{~m}^{2}$ or more.

We hypothesized that there would be a relationship between the size of the largest patch on a property, average density of European chafer larvae in the patch, and the average density of European chafer throughout the entire property. We used the number of contiguous sample locations with one or more grubs as an estimate of patch size. If the hypothesized relationship existed, then average grub density from throughout a site could be used to predict whether there was a patch of grubs with average density in excess of one per plug. Use of average density from throughout a property as a decision criterion would allow application of well-developed sampling tools. The only alternative would be extensive mapping of grub density at a site; an impractical alternative. To determine whether a relationship existed between patches of grubs and average density of grubs, we plotted patch size $(y)$ against average density $(x)$ and used the size of the symbol plotted for each data point to represent density in the patch.

Risk Rating Model. The data on site characteristics were used in a forward stepping analysis of variance (SYSTAT 1992) to determine whether there was a relationship between some or all of these variables and average grub density per plug. Factors were added to the model based on an $F$ value with $P \leq 0.05$. The forward-stepping procedure identified age of the lawn, percentage of shade, and percentage of Kentucky bluegrass as significant predictors. A new model consisting of these factors and first-level interactions was then fit to the data again using the forward-stepping procedure. None of the interactions were significant: Based on these results, we constructed a risk rating system wherein the age of the lawn, percentage of shade, and percentage of Kentucky bluegrass were used to classify properties into two groups; those that would not be sampled and not treated and those where a treatment decision would be based on the outcome of sampling.

Sampling Experiments. Counts of European chafer larvae were used to compute means $(m)$ and variances $\left(s^{2}\right)$ for each property. These estimates were then fit to the model $s^{2}=a \mathrm{mb}$ (Taylor 1961) by using logarithms to linearize the model and estimating parameters by way of linear regression. Data from 38 properties were used to determine whether counts of grubs in $11-\mathrm{cm}$ plugs could be described by Poisson or negative binomial distributions. These 38 data sets were used because at least 100 samples were taken at each of these properties and they represented a wide range of grub densities. The computer program Discrete (Gates et al. 1987) was used to fit the data by way of maximum likelihood to the models. A chi-square test $(\alpha=0.05)$ was used to measure significant departure of the counts from the two discrete probability models.

Control Decision Rules. Sampling procedures used to determine whether a pest population requires control usually classify density with respect to a threshold value and most often are sequential (Kuno 1991, Binns \& Nyrop 1992). Two deficiencies of sequential classification procedures may obviate their use. First, because density can be classified and sampling curtailed after each sample is counted, there is no guarantee that samples from throughout a particular site will be taken before sampling is terminated. Second, determination of the performance of sequential procedures requires that sample counts can be described by probability distribution models and such models can not always be fit to the data. The need to describe sample counts using probability distribution models was not an obstacle in this case; however; when sampling European chafer larvae, we felt it imperative that a representative sample be taken from throughout a property before density was classified. Because of this need we devised a sampling protocol based on double sampling (Nyrop \& Wright 1.985).

To construct a double sampling plan two hypotheses concerning mean density $(m)$ and a threshold value $(T)$ are constructed: $H_{0}: m \leq T$ and $H_{1}: m>T$. A sample of $n_{1}$ observations is taken. If the mean $\left(m_{1}\right)$ of this sample is less than or equal to some $A, H_{0}$ is accepted. $A$ satisfies the condition $A<T$. If $m_{1} \geq R, H_{0}$ is rejected. $R$ satisfies the condition $R>T$. The mean is compared with $A$ and $R$ as opposed to $T$ to guard against incorrect classification of the population when the sample size is $n_{1}$. If $A<m_{1}<R$, another sample of $n_{2}$ observations is taken and a mean computed. If $\left[\left(n_{1} m_{1}+n_{2} m_{2}\right) /\left(n_{1}+n_{2}\right)\right] \leq T, H_{0}$ is accepted. Otherwise, $H_{0}$ is rejected.

Performance of any sampling plan that classifies density with regard to a threshold is judged by the operating characteristic $(O C)$ and average sample 
number functions (ASN). The OC specifies the probability of accepting the null hypothesis given any true mean and the ASN the expected number of samples required to reach a decision. $O C$ and ASN functions for double sampling plans can be computed analytically (Nyrop \& Wright 1985) or using simulation (Nyrop \& Binns 1991). We estimated these functions by way of simulation by using a negative binomial distribution with variable $k$ to model sampling. We set $n_{1}$ and $n_{2}$ to 20 and $A$ and $R$ were determined as $90 \%$ confidence half limits about the threshold $T$ when a sample size of 20 was used. A minimum sample size of 20 was selected because we felt this would be the minimum number needed to obtain a representative sample from most home lawns. If a second sample was required, an additional batch of 20 plugs was specified because we felt it would be just as important to take the second set of samples from throughout the property before classifying density. Ninety percent confidence intervals were used for $A$ and $R$ because we felt acceptable $O C$ and AS.N functions were produced using these values. The variance was modeled as a function of the mean using Taylor's (1961) variance-mean model and $k$ of the negative binomial distribution was then specified as $m^{2} /\left(s^{2}-m\right)$. Estimates of OC and ASN functions were based on simulating sampling 1,000 times for a range of mean densities.

The plot of patch size against site-wide density indicated a threshold density should be in the range of $0.1-0.35$ grubs per $11-\mathrm{cm}$ plug. However, it was not clear from these data what the threshold should actually be. Therefore, we constructed sampling plans for thresholds of $0.1-0.35$ in increments of 0.05 . As an aid in selecting a threshold and associated sampling plan to use, we developed two error functions that we call type a and type $b$ errors. Type a error describes the proportion of sites for which a decision to treat should have been made but was not and type $b$ error describes the proportion of sites for which a decision to not treat should have been made but was not. Both errors are expressed in terms of site-wide mean density. Recall that properties requiring treatment are those for which there was a patch of grubs with an average density of one grub per plug over at least $27 \mathrm{~m}^{2}$ of contiguous area.

Type a and type b errors are computed from the OC function and an empirically derived function that describes the proportions of sites requiring control (PCR) in relation to site-wide mean density. Another way of interpreting the PRC is as the probability of a site harboring a patch of grubs requiring control given the site-wide grub density. The PRC was estimated by determining the proportion of sites requiring control for intervals of property-wide mean density and then fitting a logistic function by way of a Simplex algorithm (SYSTAT 1992) to these estimates. Using the PRC and $O C$ functions and assuming independence of

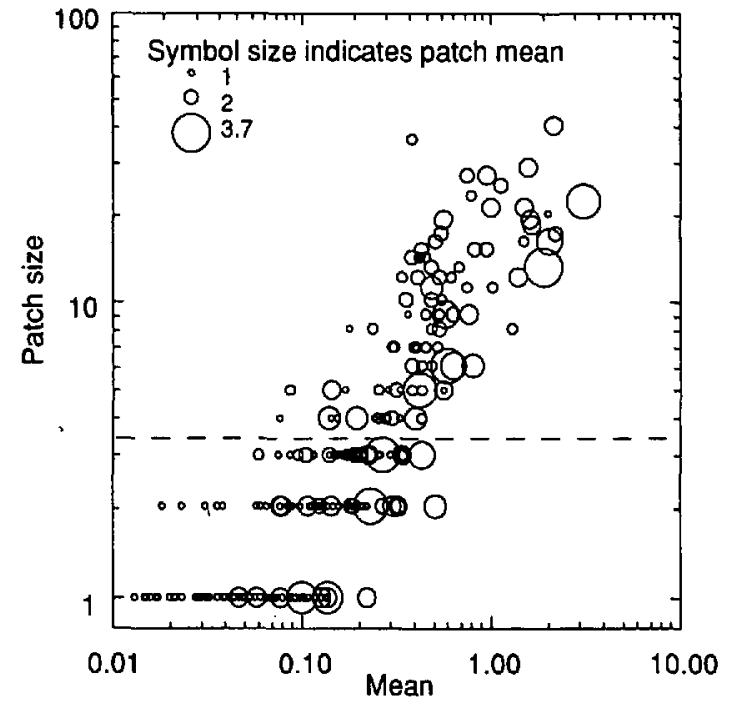

Fig. 1. Relationship between patch size ( $y$-axis), average grub density in the patch (symbol size), and average grub density over an entire property ( $x$-axis). The dashed line separates properties that require control of European chafers from those that do not.

the two, the conditional probability of treating when treatment is necessary is calculated as

$$
\operatorname{Pr}[\text { treat } \mid \text { need treat }]=[1-\mathrm{OC}] * \mathrm{PRC} \text {. }
$$

The conditional probability of not treating when no treatment is warranted is calculated as

$\operatorname{Pr}[$ no treat $\mid$ not need treat $]=O C *[1-$ PRC $] .(2)$

Using these two conditional probabilities, the type $a$ and $b$ errors are

$$
\text { ETA }=\text { PRC }-\operatorname{Pr}[\text { treat } \mid \text { need treat }]
$$

and

$$
\begin{aligned}
\text { ETB }= & {[1-\text { PRC }] } \\
& -\operatorname{Pr}[\text { no treat } \mid \text { not need treat }] .
\end{aligned}
$$

\section{Results}

Density-Patch Relationship. Patch size and European chafer larval density in the patch increased as site-wide grub density increased with the patch size-density relationship being most pronounced (Fig. 1). Thus, mean density over the entire site might be used to predict whether a property is likely to harbor a patch of European chafer larvae that requires control. The tentative nature of this conclusion stems from the need to juxtapose the pattern evinced in Fig. 1 with OC functions for sampling plans that might be used. This will be discussed shortly.

The properties shown in Fig. 1 are separated by a dashed line into those on which European chafer required control $(29.2 \%)$ and those where no control was needed. Recall that this distinction is 

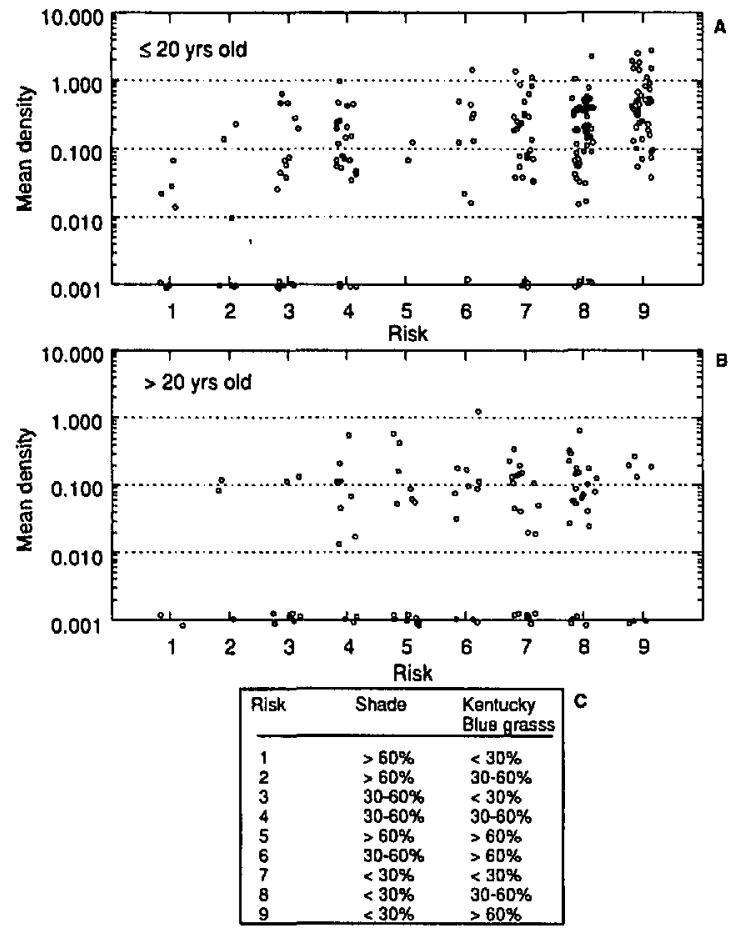

Fig. 2. Densities of European chafer larvae on properties assigned risk values of 1 through 9 and having lawns $\leq 20 \mathrm{yr}$ old $(A)$ and $>20 \mathrm{yr}$ old (B). Levels of shade and Kentucky bluegrass corresponding to each of the nine risk levels (C).

based on properties having a patch size of four or more. There is no site-wide density that clearly separates properties into those where intervention is needed and those where no control is warranted. All that can be said at this point is that a threshold must lie in the range of $0.1-0.35$ grubs per $11-\mathrm{cm}$ plug. A threshold $>0.35$ would allow too many sites with large patches of European chafer larvae to go untreated and a threshold $<0.1$ would result in too many properties receiving unnecessary insecticide treatments. We will return to the question of selecting a threshold after presenting OC functions for prospective sampling plans.

Risk Rating Model. The site factor model explained $21 \%$ of the variation in site-wide European chafer larval density. Site characteristics of age $(F$ $=20.26, \mathrm{df}=1,296)$, percentage of shading $(F=$ 9.56, $\mathrm{df}=2,296)$, and percentage of Kentucky bluegrass $(F=18.22, \mathrm{df}=2,296)$ were identified as significant predictors of grub density. Because there were no interactions among the three factors, we constructed a risk classification for each of the two age groups based on the ordering of mean density for each combination of percentage of shade and percentage of Kentucky bluegrass (nine levels). The levels of shade and Kentucky bluegrass for each of the risk categories one through nine are provided in Fig. 2. Also shown in this figure are mean densities of European chafer larvae on

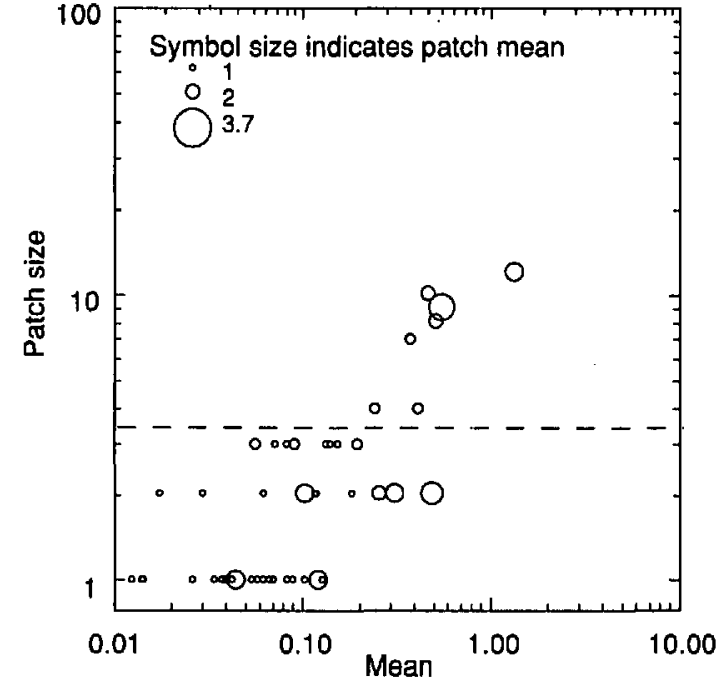

Fig. 3. European chafer grub populations on properties excluded from sampling based on risk assessment. Thirty-eight properties on which no grubs were found were not plotted. The dashed lines separates properties into a group that should be treated (above the line) and a group that should not be treated.

sites identified according to the two age classes and nine risk levels. The densities shown have 0.001 . added to them to allow plotting on a logarithmic scale and these values were jittered (SYSTAT 1992) to avoid very similar values from being overlayed.

As stated previously, an action threshold for European chafer grubs must be in the interval of 0.10.35 larvae per $11-\mathrm{cm}$ plug. With this range in mind, we chose risk values of $1-3$ for lawns $\leq 20$ $\mathrm{yr}$ in age and $1-4$ for lawns older than $20 \mathrm{yr}$ to signal that no sampling need be done. The properties that were excluded from sampling based on this risk assessment and that had densities greater than zero are shown in Fig. 3. Only 7 properties of $315(2 \%)$ were erroneously classified. However, use of the risk rating system would have obviated the need for sampling on $26 \%$ of all the properties. It is important to note that not all high risk sites have high density European chafer populations so one can not infer that all high risk sites should be treated.

Sampling Experiments. Taylor's (1961) variance-mean model provided a good description of the relationship between the variance and mean of European chafer larval counts $(\ln a=0.245, \mathrm{SE}=$ $0.04 ; b=1.056, \mathrm{SE}=0.018$, mean square error $\left.=0.113, r^{2}=0.929\right)$. When fit to Poisson and negative binomial probability distribution models, 10 of the 38 data sets used in the analysis were described by a Poisson model, 12 by a negative binomial model, and 11 were equally well described by both Poisson and negative binomial models. Five data sets could not be described by either the Poisson or negative binomial distributions. We attempted to fit positive binomial and Neyman type 

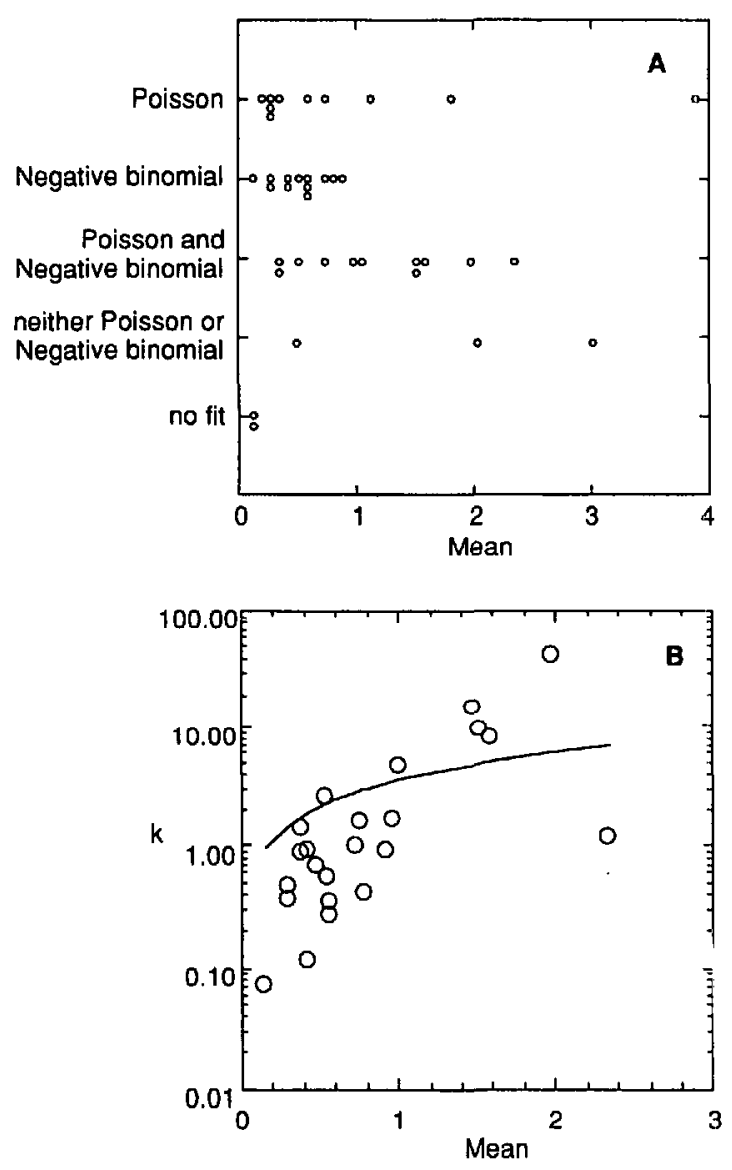

Fig. 4. Distribution of means for sets of European chafer grub counts fit to negative binomial and Poisson distribution models (A). Maximum likelihood estimates of $k$ for the negative binomial distribution (circles) and values predicted using a variance-mean model (line) (B).

A distributions to these sets of counts. Neither of these two models could be fit to two of these five data sets. One of the remaining three data sets were described by the positive binomial and two by Neyman type A distributions. It should be noted that the Neyman type A could be used to describe 24 of the 38 data sets; however, the Poisson or negative binomial models are preferred because they are easier to work with. There was no discernible pattern in the means for data sets described by the different probability models (Fig. $4 \mathrm{~A})$.

We selected the negative binomial distribution to describe counts of European chafer larvae in 11$\mathrm{cm}$ plug samples instead of the Poisson distribution because variances for the negative binomial distribution are larger. As a result, simulations in which random variables are generated according to a negative binomial distribution and are used to measure the performance of sampling plans will indicate that the plans are less precise than if simulations were based on the Poisson distribution.
We felt use of the Poisson distribution in the simulations would lead to an overly sanguine picture of errors that may result from use of the sampling plans or unrealistic estimates of required sample sizes.

The parameter $k$ of the negative binomial distribution often shows dependency with the mean, and this was the case for the European chafer grub counts. As a result, simulations of sampling should incorporate changes in $k$ due the mean. Taylor's variance-mean model can be used for this purpose by computing the variance as a function of the mean and then computing $k$ using the method of moments. Maximum likelihood estimates of $k$ obtained from the data are portrayed in Fig. 4B along with $k$ calculated using the variance-mean model. At low densities, predicted values of $k$ were greater than the maximum likelihood estimates, whereas at higher densities predicted vahes of $k$ were less than the maximum likelihood estimates.

Discrepancies between predicted and estimated values of $k$ should have at most minimal effect on the estimated performance of the sampling plans. This is because we have used a double sampling protocol with a sample size of 20 for each batch of samples that are processed. With this sample size, the central limit theorem can be invoked and the sum of grubs found will be approximately normally distributed. Therefore, the precise distribution of individual observations is less important than if a fully sequential sampling plan was being evaluated.

Control Decision Rules. Stop boundaries for the double sampling plans were calculated as

$$
A=T-\sqrt{\frac{1.277 * T^{1.056}}{20}} * 1.65
$$

and

$$
R=T+\sqrt{\frac{1.277 * T^{1.056}}{20}} * 1.65
$$

The OC and ASN functions for sampling plans with thresholds $(T)$ of $0.1-0.35$ grubs per plug are illustrated in Fig. 5. The expected number of samples required to reach a decision are near the maximum for densities close to the thresholds. Nonetheless, the double sample plans would save sampling resources compared with always using a fixed sample size of 40 . Also shown in the figure with the OC functions are the empirically derived proportions of sites that require control as well as the PRC function fit to these data. Recall that sites deemed requiring control have patches of grubs with a density of at least one per $11-\mathrm{cm}$ plug over a minimal area of $27 \mathrm{~m}^{2}$.

The OC and PCR functions were used to calculate type a (proportion of sites requiring control and not treated) and type $b$ (proportion of sites needlessly treated) errors (Fig. 6). For the thresholds used, type a errors are always greater than type b errors. Type a errors increase as the thresh- 

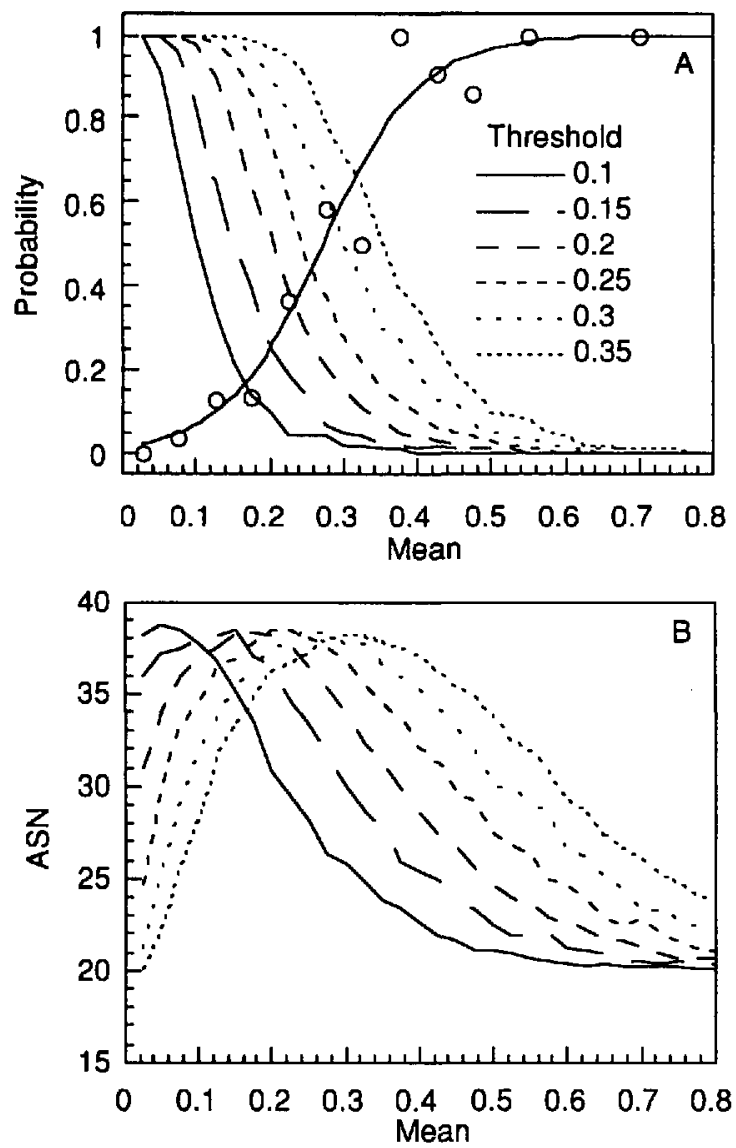

Fig. 5. Operating characteristic (OC) functions for four double sample plans and the proportion of sites requiring control $(A)$. The fitted line is calculated as $1 /[1+$ $\exp (4.089-15.029 x)]$. Average sample number (ASN) functions for four double sample plans (B).

old increases and type b errors decrease with increasing threshold values.

To interpret fully the influence of these error rates on decision rule performance, it is helpful to know the likelihood that a particular grub density will be encountered at a site. For example, type a and $b$ errors are maximum for densities close to the threshold. This result is more important if many sites that might be sampled are likely to have European chafer larval densities close to the threshold values and the result is less significant if grub densities close to the threshold are rather unlikely. We used data from the 317 properties sampled to estimate the likelihood of various intervals of site-wide grub densities and expressed these results as a histogram (Fig. 6).

A risk averse manager might choose a sampling plan based on a low threshold to reduce type a errors and a manager averse to applying pesticides needlessly might choose a sampling plan based on a high threshold. We opted to approximately balance type $a$ and $b$ errors and recommend use of $a$
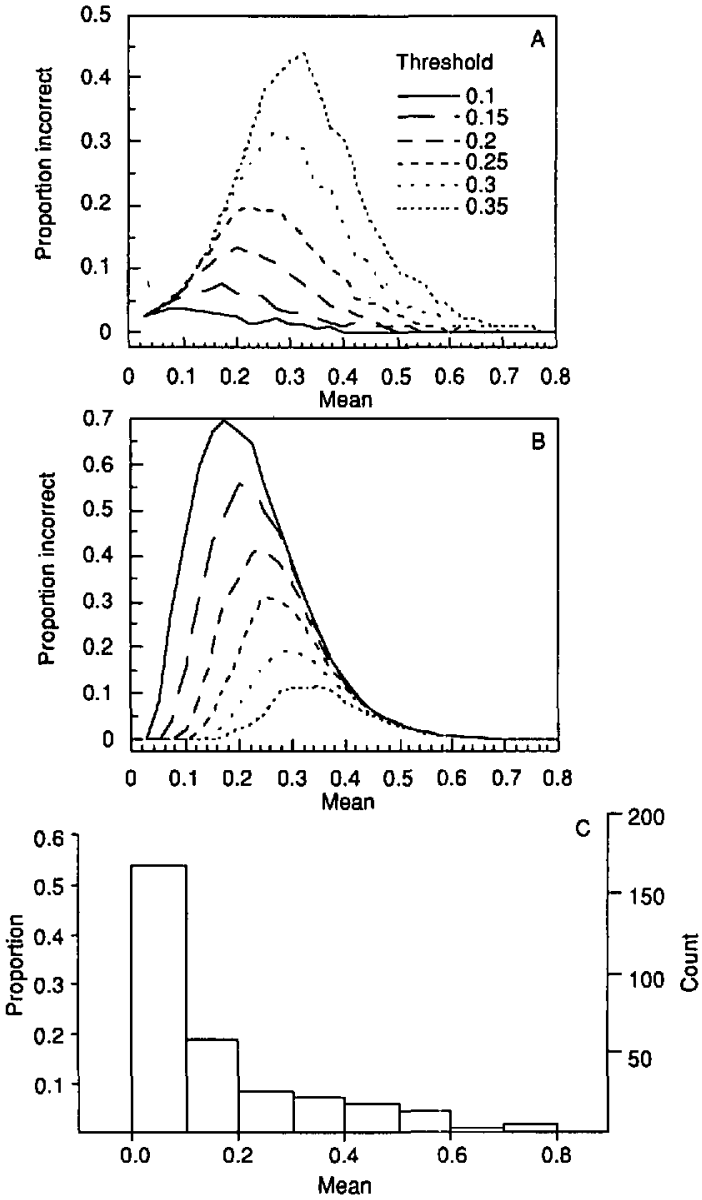

Fig. 6. Type $a(A)$ and type $b(B)$ errors for six double sample plans used to classify European chafer populations with regards to a threshold and the distribution of sample means for 317 properties simpled (C).

threshold equal to 0.25 grubs per $11-\mathrm{cm}$ plug. For this threshold, the maximum type a and type $b$ errors are $\approx 0.2$ and 0.3 , respectively. However, these relatively large error rates occur over a narrow range of mean densities. Furthermore, assuming that the distribution of mean densities that we recorded in 1990 are representative of other years and other areas, the mean densities for which maximum error rates occur are not exceedingly likely.

\section{Discussion}

In this article we developed and analyzed the performance of a control decision rule for European chafer larvae infesting residential turf lawns. The decision rule functions in two steps. First, the age of the lawn, percentage of shading, and the percentage of the turf that is Kentucky bluegrass is determined. Based on these estimates, the site is classified as either requiring no sampling and no treatment or the site should be sampled and a treatment decision based on the outcome of sam- 
pling. Provided the data used to develop the risk assessment system are indicative of patterns that will be found at other times and locations, using this system can save sampling resources and the likelihood of erroneously categorizing the site as not needing sampling or control is low.

The risk assessment system is the most tenuous aspect of the control decision rule because data on which it is based only span a single year and single location. From a practical perspective, the risk assessment system need not even be used and all sites can always be sampled. On a more conceptual footing, our ability to devise a risk assessment protocol for incorporation in a control decision rule should catalyze further work in this area. An obvious next step is to collect more data to make the system more robust.

The second step in executing the control decision rule is to sample the population of European chafer larvae. This is done by extracting $2011-\mathrm{cm}$ plugs from throughout the site and counting the number of grubs found. If the total is $\leq 20 * A$ or $\geq 20 * R$, sampling is terminated and an appropriate decision is made. Otherwise, 20 additional plug samples are collected and the total grubs found in the two batches of plugs is compared with $40 * T$ and a decision is made.

This sampling plan is based on our expert opin. ion concerning what constitutes a patch of grubs that warrant treatment, the relationship between patch size and site-wide density, and the distribution of counts of grubs from $11-\mathrm{cm}$ plug samples. The least important of these and also the best known is the distribution of counts of grubs. This paradox is not unique to the case at hand but probably applies to most pest control decision rules. The relationship between patch size and site-wide density is crucial, the data set used to describe this relationship is large, and the pattern evinced is probably robust. It is of course possible that different environmental conditions will produce a different relationship, but we think this is unlikely. Of primary importance is what constitutes a patch of European chafer grubs that necessitates control. There are two aspects to this question. First, is an area of $\approx 30 \mathrm{~m}^{2}$ with a density of grubs putatively capable of causing economic injury indicative of the need for insecticide treatment? Second, with what precision did our data identify sites that meet this condition? Additional research will be required to answer these questions. To address the first query, property owner tolerance for different sized areas of grub damage to turf needs to be assessed. To answer the second question, grub densities must be mapped at a finer spatial scale (e.g., every meter) than was done in the study reported herein and these data used to determine whether samples spaced at intervals of $3 \mathrm{~m}$ correctly identify the existence of patches of European chafer larvae.

Despite these questions, we feel the decision rule presented here can be used effectively to schedule pesticide applications directed at European chafer larvae. The paradigm used to construct this decision rule should also prove useful for building decision rules for other soil inhabiting pests of turfgrass.

\section{References Cited}

Binns, M. R. \& J. P. Nyrop 1992. Sampling insect populations for the purpose of IPM decision making. Annu. Rev. Entomol. 37: 427-453.

Burrage, R. H. \& G. G. Gyrisco. 1954. Distribution of third-instar larvae of the European chafer and the efficiency of various sampling units for estimating their populations. J. Econ. Entomol. 47: 1009-1014.

Gates, C. E., F. G. Ethridge \& J. D. Geughan. 1987. Fitting discrete distributions. User's documentation for the FORTRAN computer program DISCRETE. Texas A\&M University, College Station.

Kuno, E. 1991. Sampling and analysis of insect populations. Annu. Rev. Entomol. 36: 285-304.

Ng, Y. G., J. R. Trout \& S. Ahmad. 1983a. Spatial distribution of the larval populations of the Japanese beetle (Coleoptera: Scarabaeidae) in turfgrass. J. Econ. Entomol. 76: 26-30.

1983b. Sequential sampling plans for larval populations of the Japanese beetle (Coleoptera: Scarabaeidae) in turfgrass. J. Econ. Entomol. 76: 251-253.

Nyrop, J. P. \& M. Binns. 1991. Quantitative methods for designing and analyzing sampling programs for use in pest management, pp. 67-132. In D. Pimentel [ed.], Handbook of pest management in agriculture, vol. III, 2nd ed. CRC, Boca Raton, FL.

Nyrop, J. P. \& R. J. Wright. 1985. The use of double sample plans in insect sampling with reference to the Colorado potato beetle Leptinotarsa decemlineata (Say). Environ. Entomol. 14: 644-649.

Shetlar, D. J., P. R. Heller \& P. D. Irish. 1990. Turfgrass insect and mite manual, 3rd ed. Pennsylvania Turfgrass Council, Bellefonte, PA.

SYSTAT. 1992. SYSTAT: statistics, version 5.2 ed. SYSTAT, Evanston, IL.

Tashiro, H. 1987. Turfgrass insects of the United States and Canada. Cornell University, Ithaca, NY.

Taylor, L. R. 1961. Aggregation, variance, and the mean. Nature (Lond.) 189: 732-735.

Received for publication 15 April 1994; accepted 5 December 1994 . 